\title{
7464
}

\section{Progression of Idiopathic Pulmonary Fibrosis in patients with past occupational exposure to dusts}

Occupation, Interstitial lung disease, Idiopathic pulmonary fibrosis

\author{
F. Liviero' ${ }^{1}$, P. Mason ${ }^{1}$, G. Guarnieri ${ }^{1}$, M. C. Scarpa ${ }^{1}$, E. Balestro ${ }^{1}$, P. Maestrelli ${ }^{1}$
}

${ }^{1}$ Department of Cardiologic, Thoracic and Vascular Sciences, University of Padova - Padova (Italy)

Background: Interstitial lung diseases with usual interstitial pneumonia (UIP) pattern include Idiopathic Pulmonary Fibrosis (IPF) and asbestosis. Despite the label of 'idiopathic' there is epidemiological evidence that IPF is associated to occupational exposures to dust.

Aims: To test the hypothesis that IPF in individuals with past occupational exposure to dust may have a slower progression of the disease as it occurs in UIP pattern due to asbestos exposure.

Methods: We collected occupational history of 44 patients with proven diagnosis of IPF. All patients had been under pirfenidone therapy for at least one year. Occupational exposure was defined when a subject had at least $10 \mathrm{yr}$ of cumulative work exposure to inorganic or organic dusts. Annual decline of Forced Vital Capacity (FVC) was used to assess functional progression during therapy. The duration of symptoms before diagnosis was determined (<1yr, rapid onset, R; >1yr slow onset, $S$ ).

Results: Sixteen/44 IPF patients (36\%) had occupational exposure to inorganic $(n=11)$ or organic $(n=5)$ dusts. IPF patients with dust exposure tended to show slower onset of symptoms ( $\mathrm{S}=64 \%$ ) compared with non-exposed group $(S=37 \%)$ [X2=2.75; $p=0.09]$. Independently from smoking habit, IPF patients with dust exposure exhibited a reduced decline in lung function compared to IPF patients without exposure, although not statistically significant (FVC-120+58 $\mathrm{ml} / \mathrm{yr}$ vs $-209+86 \mathrm{ml} / \mathrm{yr}$ ).

Conclusions: IPF patients with occupational exposure to dusts tended to have a slower onset of symptoms and less functional decline during therapy than non-exposed IPF patients.

The results suggest a possible role of dust exposure in the heterogeneity of IPF progression. 